\title{
Risk prediction for invasive candidiasis
}

\author{
Armin Ahmed, Afzal Azim, Arvind Kumar Baronia, K. Rungmei S K Marak ${ }^{1}$, Mohan Gurjar
}
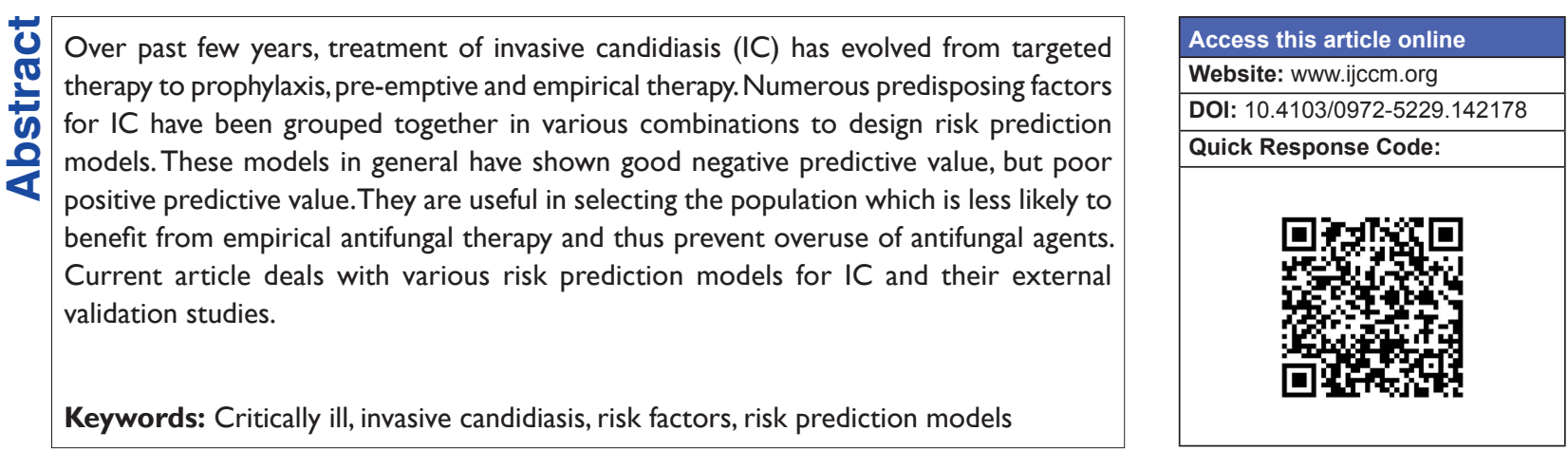

\section{Introduction}

One of the most important skills of intensive care physicians is their ability to preempt critical events while caring for severely sick individuals. In order to give a universally acceptable and scientifically sound shape to the prediction skill, various risk prediction models have been developed for a number of medical illnesses (e.g. deep venous thrombosis, community-acquired pneumonia).

Fungal infections are increasing worldwide. ${ }^{[1,2]}$ These infections are associated with high mortality, morbidity and increased cost of care ${ }^{[3,4]}$ Candida is the most common fungal pathogen in human beings. According to the 1-day point prevalence (Extended Prevalence of Infection in Intensive Care II) study conducted over 75 countries in 2007, Candida was third most common pathogen with an infection rate of $17 \%$ after Staphylococcus aureus and Pseudomonas spp. ${ }^{[5]}$ Half to one-third of Candida infections occur in Intensive Care Unit (ICU) patients. Emergence of Candida is further complicated by increased rate of infections due to non-albicans species and growing

From:

Departments of Critical Care Medicine and ${ }^{1}$ Microbiology, Sanjay Gandhi

Postgraduate Institute of Medical Sciences, Lucknow, Uttar Pradesh, India

\section{Correspondence:}

Dr. Afzal Azim, Department of Critical Care Medicine, Sanjay Gandhi Postgraduate Institute of Medical Science, Lucknow - 226 014, Uttar Pradesh, India.

E-mail: draazim2002@gmail.com resistance to antifungal agents..$^{[6,7]}$ Five common species of Candida responsible for blood stream infection are Candida albicans, Candida glabrata, Candida parapsilosis, Candida tropicalis, and Candida krusei. Other species causing Candida infections are Candida lusitaniae, Candida guilliermondii, Candida rugosa.

Candida infection is associated with excess attributable mortality of $10-49 \%$ and increase in length of hospital stay of 3-30 days. ${ }^{[8]}$ Delayed diagnosis of fungal infections is a common occurrence, and several studies have shown higher mortality associated with delayed initiation of appropriate antifungal therapy. ${ }^{[9-11]}$ At least five meta-analyses studied the role of prophylactic antifungal among surgical and critically ill patients. ${ }^{[12-16]}$ All showed successful reduction in the rate of fungal infections with the use of antifungal prophylaxis while two showed a significant reduction in total mortality. Indiscriminate use of antifungal prophylaxis can lead to the development of resistant species. Playford et al. recommended that prophylaxis should be given to a subgroup of the population where incidence of invasive candidiasis (IC) is $>10 \%$. In such a situation, the number needed to treat to prevent one infection is twenty. Therefore, it is necessary to identify those patients who are at increased risk of IC.

Colonization almost invariably precedes Candida infection and frequency of infection increases with an 
increased number of sites colonized. Besides colonization and immunosuppression, factors known to be associated with increased risk of invasive fungal infections (IFIs) are broad spectrum antibiotic use, total parenteral nutrition (TPN), central venous catheter (CVC), surgery, renal replacement therapy, diabetes, prolonged mechanical ventilation, severe sepsis, and high Acute Physiology and Chronic Health Evaluation (APACHE) II score. ${ }^{[17]}$ These risk factors have been used in various combinations to design risk prediction models [Table 1]. Current article is a review of risk prediction models developed to predict IC and external validation of these models.

\section{Review}

Literature search was done on PubMed and Medline databases from January 1990 to June 2013. Terms like "risk factors for IC," "prediction models/scores for IC," "validation of Candida score," "validation of clinical prediction rules (CPRs) for IC" were used for the search. References from the relevant articles were also searched manually by two researchers separately. Studies dealing with the development of risk prediction models for IC, as well as studies on external validation of any of the already developed model, were included for evaluation. Studies in which risk factors for IC were studied, but no model was developed or validated were excluded from our study.

A total of 14 studies met the inclusion criteria. There were total 9 derivation studies (studies in which a risk prediction model was developed) and 5 external validation studies (studies in which risk prediction models were validated). The studies were further classified as risk prediction models based on microbiological parameters only, models based on clinical parameters only, and models based on clinical as well as microbiological parameters [Tables 2, 3 and 4].

\section{Models based on microbiological parameters only Derivation study}

Pittet et al. attempted to quantify the severity of Candida colonization and hypothesized a probable link between the severity of colonization and infection. ${ }^{[18]}$ Based on culture reports of samples taken from different distinct body sites (dbs), colonization index (CI) and corrected colonization index (CCI) were developed. The study was conducted over a period of 6 months on patients

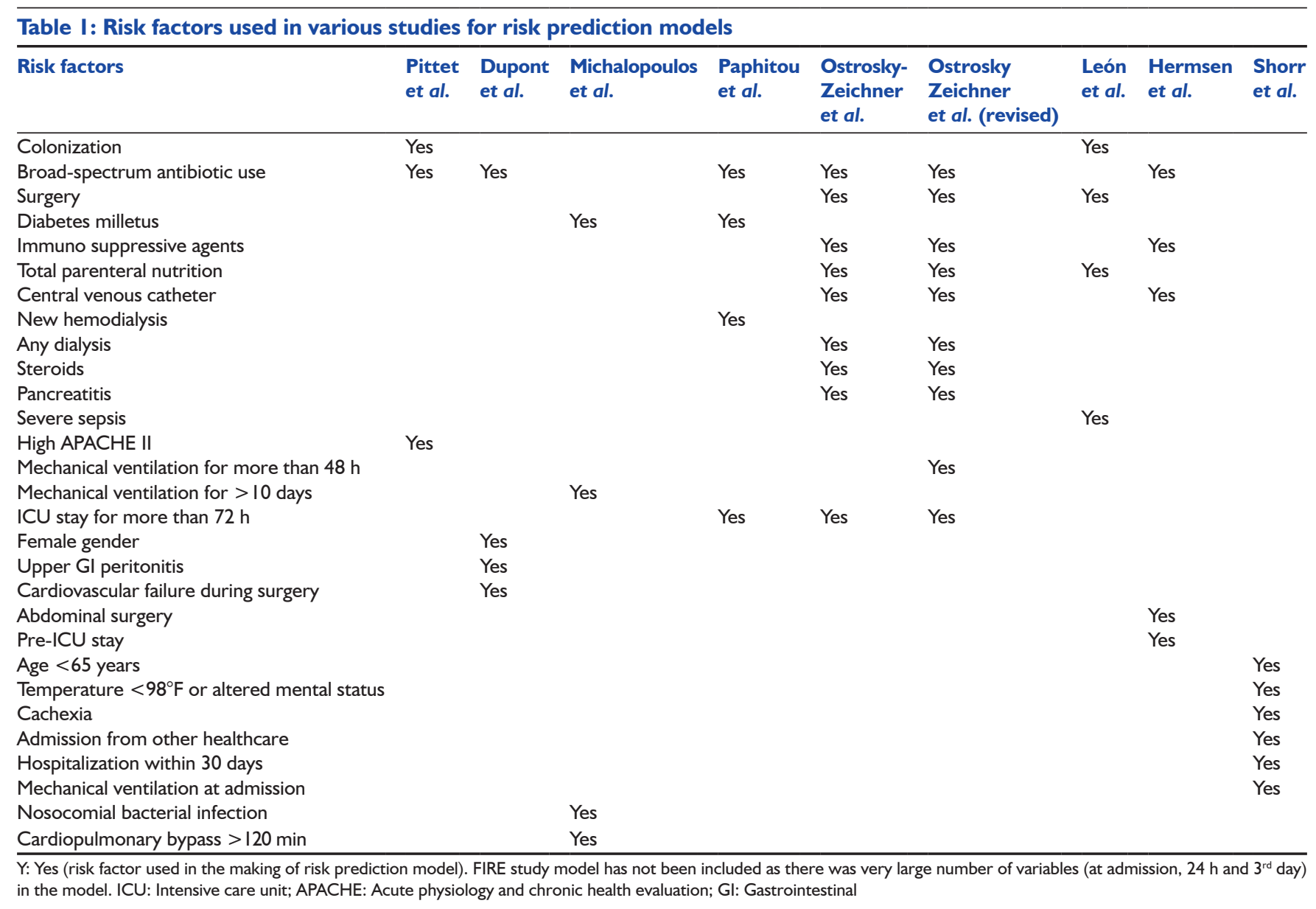




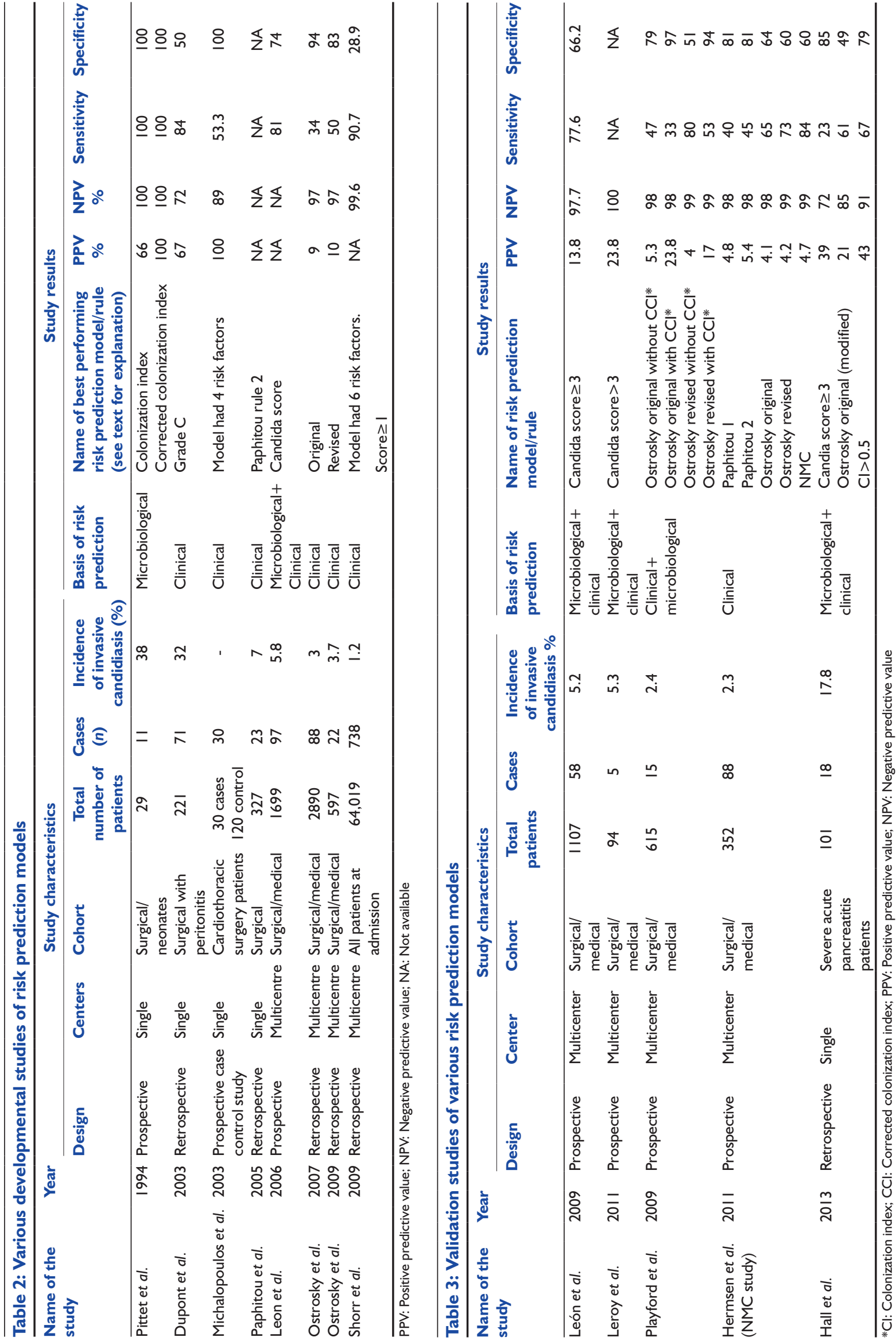


Table 4: Calculation of commonly used risk prediction scores/models for invasive candidiasis

\begin{tabular}{|c|c|c|}
\hline Risk model & Components & Cut off \\
\hline $\begin{array}{l}\text { Candida } \\
\text { score }\end{array}$ & $\begin{array}{l}\text { Parenteral nutrition-I, Surgery- I point, } \\
\text { Multifocal colonization (colonization at more } \\
\text { than I site) - I point and severe sepsis- } 2 \text { points }\end{array}$ & $\begin{array}{l}\text { Score } \geq 3 \text { is } \\
\text { considered } \\
\text { positive }\end{array}$ \\
\hline $\begin{array}{l}\text { Colonization } \\
\text { index }\end{array}$ & $\begin{array}{l}\text { Number of nonblood dbs (distinct body sites) } \\
\text { colonized by Candida spp divided by the total } \\
\text { number of body sites cultured }\end{array}$ & $\begin{array}{l}\text { Score } \geq 0.5 \text { is } \\
\text { considered } \\
\text { positive }\end{array}$ \\
\hline $\begin{array}{l}\text { Corrected } \\
\text { colonization } \\
\text { index }\end{array}$ & $\begin{array}{l}\mathrm{Cl} \text { multiplied by the ratio of heavily colonized } \\
\text { sites to total number of sites colonized }\end{array}$ & $\begin{array}{l}\text { Score } \geq 0.4 \text { is } \\
\text { considered } \\
\text { positive }\end{array}$ \\
\hline $\begin{array}{l}\text { Ostrosky } \\
\text { clinical } \\
\text { prediction } \\
\text { rule }\end{array}$ & $\begin{array}{l}\text { ICU stay X } 72 \text { hours and Mechanically } \\
\text { ventilated } X 48 \text { hours and Antibacterial } \\
\text { antibiotic use } X \text { day I-3 and CVC X day I-3 } \\
\text { AND at least one of the following } \\
\text { Any surgery, day }-7-0 \\
\text { Immunosuppressive use, day }-7-0 \\
\text { Pancreatitis, day }-7-0 \\
\text { TPN, day I-3 } \\
\text { Any dialysis, day I-3 } \\
\text { Steroid use, day }-7-0\end{array}$ & \\
\hline
\end{tabular}

ICU: Intensive care unit; CVC: Central venous catheter; TPN: Total parenteral nutrition; $\mathrm{Cl}$ : Colonization index

admitted in surgical and neonatal ICU. All patients found to have significant colonization in surveillance cultures were included in the study. Colonization was defined as isolation of Candida from three or more samples from same or different sites for two or more consecutive screening days. Infection was defined as patient having candidemia or severe Candida species infection requiring use of antifungal therapy. Patients were divided into two groups, infected $(n=11)$ and colonized $(n=18)$. The infected group had three characters significantly different from the colonized group, namely higher APACHE II score at the time of admission, longer duration of antibiotic exposure and increased intensity of Candida colonization. Out of three variables, APACHE II and intensity of colonization proved to be an independent predictor of Candida infection in logistic regression analysis. They defined $\mathrm{CI}$ as nonblood dbs sites colonized by Candida divided by total number of sites tested and CCI as CI multiplied by ratio of heavy growth dbs upon the total number of dbs positive for Candida and proposed these indices as method of assessing severity of colonization. CI in the infected group was 0.70 as compared to 0.47 in the colonized group $(P<0.01)$. Mean CCI of the colonized group was 0.16 while that of the infected group was $0.56(P<0.01)$. A cut off value of $\mathrm{CI}>0.5$ and $\mathrm{CCI}>0.4$ identified all infected patients.

These indices (CI and CCI) have never been validated in robustly designed studies particularly in nonsurgical patients. Though clinicians do tend to consider multiple site colonization with Candida, an important risk factor for the development of IC, such type of surveillance is difficult and therefore various researchers attempted to design prediction rules only on the basis of clinical risk factors.

\section{External validation}

Colonization index and CCI have never been validated in large randomized control trials. There are at least 10 studies dealing with $\mathrm{CI}$ and $\mathrm{CCI}$, but none qualified the inclusion criteria for our study and are not discussed here.

\section{Models based on clinical parameters (clinical prediction rules) \\ Derivation studies}

Dupont et al. conducted a study to identify risk factors for isolation of fungus (yeast) from peritoneal fluid in surgical patients. ${ }^{[19]}$ The risk score was generated by retrospective study of a cohort of 221 patients and prospectively validated on 57 patients in the same ICU. They identified four risk factors (cardiovascular failure, upper gastrointestinal tract origin for peritonitis, female gender, and previous antimicrobial therapy) independently associated with isolation of Candida from peritoneal fluid. Four grades of the score were formed (grade A, B, C, D). Grade A = no risk factor/one risk factor, grade $\mathrm{B}=2$ risk factors, grade $C=3$ risk factors, grade $D=4$ risk factors. Grade C (presence of at least three risk factors) was considered to have the best overall accuracy with sensitivity $84 \%$ and specificity $50 \%$. The prediction rule was made only for secondary peritonitis patients and hence cannot be generalized in other population group.

Michalopoulos et al. studied risk factors for candidemia on cardiothoracic ICU patients in case-control study (30 candidemia patients and 120 control patients). ${ }^{[20]}$ They proposed a model based on four risk factors found to be independently associated with candidemia in stepwise logistic regression (mechanical ventilation for $>10$ days, nosocomial bacterial infection and/or bacteremia, cardiopulmonary bypass duration $>120 \mathrm{~min}$ and diabetes mellitus). The model had a sensitivity of $53.3 \%$, specificity of $100 \%$, (positive predictive value [PPV]) PPV of $100 \%$ and (negative predictive value $[\mathrm{NPV}])$ NPV of $89 \%$.

The model was validated by the same group at two centers. There were 19 candidemia patients in the validation study. The model performed well in the validation study (sensitivity $57.9 \%$, specificity $100 \%$, PPV 100\%, NPV 99.6\%). 
Paphitou et al. developed prediction rule for surgical patients. ${ }^{[21]}$ It was a single center retrospective study conducted on 327 nonneutropenic patients staying for $>4$ days in ICU. Total percentage of IC was $11 \%$ (36 cases) which included $2.8 \%$ proven, $4.3 \%$ probable and possible $3.9 \%$ cases. Various risk factors identified by multivariate analysis were starting of new hemodialysis, diabetes mellitus and use of broad spectrum antibiotics. On the basis of these findings, three CPRs were developed and compared. The best-performing rule (referred as Paphitou rule 2 in this manuscript) was described as ICU stay equal to or more than 4 days and no antifungal use from day 7 to 3 and any of the following: Diabetes or TPN prior to ICU entry or new onset hemodialysis, or broad spectrum antibiotic use. Paphitou rule 1 was same as rule 2 except for the exclusion of broad spectrum antibiotic use.

Ostrosky-Zeichner et al. conducted a retrospective multicenter study on 2,890 patients from 12 medical and surgical ICUs in USA and Brazil. ${ }^{[22]}$ There were 88 cases of IC out of which 84 were proven, and 4 were probable. The incidence of IC the study population was 3.0\%. Two groups namely; training group and validation group were formed with $75 \%$ and $25 \%$ samples respectively. Various risk factors for IC identified in were grouped together in different combinations to form a number of CPRs. The performance of these CPRs was tested using sensitivity, specificity, PPV, NPV. The best performing rule (referred as Ostrosky original CPR in this manuscript) had a capture rate of $45.5 \%$ cases. It included following criteria; antibiotic use or CVC use and at least two of additional risk factor (surgery, immunosuppressive use, pancreatitis, TPN, dialysis and steroid use).

In 2009, they published another study after comparing modifications of the above-described rule. ${ }^{[23]}$ This was also a large multicenter retrospective study conducted on 597 patients. The incidence of IC in the study population was $3.7 \%$. In this study the best performing CPR (referred as Ostrosky revised CPR in this manuscript) was described as mechanical ventilation for least $48 \mathrm{~h}$ and antibiotic use and CVC and at least one of the following additional risk factors: Any surgery, immunosuppressive use, pancreatitis, TPN, any dialysis, steroid use. This rule had PPV of $10 \%$ NPV $97 \%$, specificity of $83 \%$ and sensitivity of $50 \%$.

Shorr et al. developed a model to identify patients with bloodstream infection likely to be caused by Candida spp. at hospital admission. ${ }^{[2]}$ They used a very large cohort (64,019 patients) from 176 acute care hospitals of United States. Rate of candidemia was $1.2 \%$ (738 patients). Six risk factors were identified as best discriminator of candidemia at admission (age $<65$ years, temperature $<98^{\circ} \mathrm{F}$ or altered mental status, previous hospitalization within 30 days, mechanical ventilation at admission, cachexia and admission from other healthcare facility). The rates of candidemia increased from $0.4 \%$ to $27.3 \%$ as the number of risk factors increased from 0 to 6. A score $>1$ had a sensitivity of $90.7 \%$ and specificity of $28.9 \%$. In the same study, this model was validated on a cohort of 24,685 patients with similar findings. Area under receiver operator curves (AUROCs) of derivation cohort and validation cohort was 0.70 and 0.71 respectively.

Harrison et al. developed and internally validated risk prediction model for IC in a multicenter study conducted in England, Wales and Northern Ireland. ${ }^{[25]}$ Out of 60,778 patients studied 383 (0.6\%) were admitted with or developed invasive fungal disease. The model was calculated at admission, at $24 \mathrm{~h}$ and at 3 days. Different variables were used for admission, $24 \mathrm{~h}$ and day 3 calculations. They used C-index (equivalent to the area under receiver operating characteristic curve) to assess the discriminatory power of the model. C-index at admission was 0.705 which improved to 0.823 at $24 \mathrm{~h}$ and 0.835 at the end of 3 days. The performance of these models dropped in validation samples (C-index 0.655, 0.732 and 0.709 for the three models). It was worst when applied to different geographical setting.

\section{External validation studies}

External validation of Paphitou and Ostrosky CPRs was done by Hermsen et al. (NMC Nebraska study). ${ }^{[26]}$ Playford et al. did external validation of Ostrosky original and Ostrosky revised CPRs in 2009. ${ }^{[27]}$

Playford et al. conducted a prospective multicenter study for external validation of Ostrosky original and Ostrosky revised CPRs with and without the addition of $\mathrm{CI}$ and CCI. The study demonstrated an improvement in PPV after addition of CCI to Ostrosky CPRs (from $5.3 \%$ to $23.8 \%$ for Ostrosky original and from $4 \%$ to $17 \%$ for Ostrosky revised). They recommended addition of colonization to the clinical risk factors for better performance of the CPRs.

Hermsen et al. conducted external validation study to compare Paphitou and Ostrosky's CPR. The success of CPR was assessed in the cohort which was divided into case and control group in 1:3 ratios. They also developed their own prediction rule named on the institute (NMC rule). The risk factors showing statistical significance 
in the study were different from the ones described in original two studies. New factors like abdominal surgery and pre-ICU length of stay were found to be significantly related to IC while pancreatitis, surgery, diabetes and hemodialysis were not shown to be statistically significant. This difference could be because of the difference in study population. The study showed poor PPV for both Paphitou and Ostrosky rule which was between $4.1 \%$ and $5.4 \%$. On the contrary, NPV was high for all the rules (>98\%). This shows that CPRs can be helpful in excluding the patient who are not likely to benefit from antifungal therapy rather than selecting the group who will benefit from such therapy.

The CPRs performed less well in validation studies as compared to derivation studies. The difference in results could be due to the difference in the study population and clinical practices. The PPV was $<5 \%$ in all studies while NPV was $>97 \%$. Therefore, present literature supports the use of CPR to identify patients who are not likely to benefit from antifungal therapy.

\section{Models based on the basis of both microbiological and clinical parameters \\ Derivation study}

León et al. 2006 conducted a prospective multicenter study on 1,699 critically ill ICU patients and developed Candida score. ${ }^{[28]}$ Patients were divided into three groups, noncolonized noninfected $(n=719)$, colonized with Candida species $(n=883)$ and proven Candida infection $(n=97)$. Mortality rates were higher in patients with multifocal colonization $(50.9 \%)$ and proven candidiasis (57.7\%) as compared to $33.2 \%$ in noncolonized noninfected group. Risk factors independently associated with proven Candida infection as found by multivariate analysis were surgery on ICU admission, TPN, severe sepsis and multifocal colonization. For calculating Candida score, each risk factor was given one point except for sepsis which was given two points. A score above 2.5 had a sensitivity of $81 \%$ and specificity of $74 \%$ in predicting Candida infection.

\section{External validation studies}

León et al. conducted external validations study on 1,107 nonneutropenic ICU patients. ${ }^{[29]}$ The sensitivity of Candida score $>3$ was $77.6 \%$, and specificity was $66.2 \%$. They also studied utility of beta-D-glucan as a diagnostic tool for IC. A cut off value of $75 \mathrm{pg} / \mathrm{ml}$ had a sensitivity of $77.8 \%$, however, the specificity was low $(52.7 \%)$. A summary of above described studies is given in Tables 2 and 3.
Leroy et al. conducted external validation of Candida score in a prospective, observational multicenter study conducted in five ICUs of France. ${ }^{[30]}$ Total 94 patients were enrolled. Rate of IC was $5.3 \%$. They found a significant association between rising Candida score and IC $(P<0.0001)$. They reported a PPV of $23.8 \%$ and NPV of $100 \%$ for a Candida score $>3$. Note that the cutoff used in this study was $>3$ rather than $>3$ as used in external validation study done by León et al.

Hall et al. conducted a single center retrospective study on 101 patients of severe acute pancreatitis. ${ }^{[31]}$ Rate of candidal infection was $17.8 \%$. Out of three (CI, Ostrosky $\mathrm{CPR}$ and Candida score) risk prediction models compared CI showed the best discrimination power (area under receiver operating curve of 0.79 ).

\section{Conclusion}

Many diverse clinical conditions have been found to be associated with risk of IC. Based on these risk factors risk prediction models have been designed. Models which include clinical as well as microbiological parameters for identifying high-risk group perform better as compared to models using clinical parameters alone. These models should be judiciously used for identifying the high-risk group and early initiation of antifungal therapy. Waiting for a positive culture report can result in a delay in initiation of therapy and increased morbidity and mortality. Issues regarding the present literature on IC are following; First many diverse clinical conditions have been found to be significantly associated with risk of IFI. These conditions have not been uniformly studied.

Second, a particular risk prediction model will be applicable only if the cohort is similar to that on which derivation study was conducted. Model developed in a particular cohort cannot be applied to all patients. Geographical variation, temporal variation, difference in antibiotic prescription policies, virulence of the most commonly colonizing Candida species etc., are some of the factors that can affect the model performance.

Third, broad spectrum antibiotic use and sepsis are important risk factors for IC. These factors are commonly present in many ICU patients making it difficult to design models solely based on clinical parameters. Further research should be done on models based on clinical as well as microbiological parameters (biomarkers and colonization). Fourth, most of the risk prediction models have high NPV and poor PPV. They are useful in identifying the patients less likely to benefit from antifungal therapy and thus restrict irrational use of antifungal agents. 


\section{Acknowledgment}

We are grateful to Reema Yadav and Preeti for their support in preparing the manuscript.

\section{References}

1. Wisplinghoff H, Bischoff T, Tallent SM, Seifert H, Wenzel RP, Edmond MB. Nosocomial bloodstream infections in US hospitals: Analysis of 24,179 cases from a prospective nationwide surveillance study. Clin Infect Dis 2004;39:309-17.

2. Rodloff C, Koch D, Schaumann R. Epidemiology and antifungal resistance in invasive candidiasis. Eur J Med Res 2011;16:187-95.

3. Rentz AM, Halpern MT, Bowden R. The impact of candidemia on length of hospital stay, outcome, and overall cost of illness. Clin Infect Dis 1998;27:781-8.

4. Smith PB, Morgan J, Benjamin JD, Fridkin SK, Sanza LT, Harrison LH, et al. Excess costs of hospital care associated with neonatal candidemia. Pediatr Infect Dis .J 2007;26:197-200.

5. Vincent JL, Rello J, Marshall J, Silva E, Anzueto A, Martin CD, et al. International study of the prevalence and outcomes of infection in intensive care units. JAMA 2009;302:2323-9

6. Montagna MT, Caggiano G, Lovero G, De Giglio O, Coretti C, Cuna T, et al. Epidemiology of invasive fungal infections in the intensive care unit: Results of a multicenter Italian survey (AURORA Project). Infection 2013;41:645-53.

7. Pfaller MA, Moet GJ, Messer SA, Jones RN, Castanheira M. Geographic variations in species distribution and echinocandin and azole antifungal resistance rates among Candida bloodstream infection isolates: Report from the SENTRY Antimicrobial Surveillance Program (2008 to 2009). J Clin Microbiol 2011;49:396-9.

8. Zaoutis TE, Argon J, Chu J, Berlin JA, Walsh TJ, Feudtner C. The epidemiology and attributable outcomes of candidemia in adults and children hospitalized in the United States: A propensity analysis. Clin Infect Dis 2005;41:1232-9.

9. Morrell M, Fraser VJ, Kollef MH. Delaying the empiric treatment of Candida bloodstream infection until positive blood culture results are obtained: A potential risk factor for hospital mortality. Antimicrob Agents Chemother 2005;49:3640-5.

10. Garey KW, Rege M, Pai MP, Mingo DE, Suda KJ, Turpin RS, et al. Time to initiation of fluconazole therapy impacts mortality in patients with candidemia: A multi-institutional study. Clin Infect Dis 2006;43:25-31.

11. Parkins MD, Sabuda DM, Elsayed S, Laupland KB. Adequacy of empirical antifungal therapy and effect on outcome among patients with invasive Candida species infections. J Antimicrob Chemother 2007;60:613-8.

12. Shorr AF, Chung K, Jackson WL, Waterman PE, Kollef MH. Fluconazole prophylaxis in critically ill surgical patients: A meta-analysis. Crit Care Med 2005;33:1928-35.

13. Cruciani M, de Lalla F, Mengoli C. Prophylaxis of Candida infections in adult trauma and surgical intensive care patients: A systematic review and meta-analysis. Intensive Care Med 2005;31:1479-87.

14. Playford EG, Webster AC, Sorrell TC, Craig JC. Antifungal agents for preventing fungal infections in non-neutropenic critically ill and surgical patients: Systematic review and meta-analysis of randomized clinical trials. J Antimicrob Chemother 2006;57:628-38.

15. Vardakas KZ, Samonis G, Michalopoulos A, Soteriades ES, Falagas ME. Antifungal prophylaxis with azoles in high-risk, surgical intensive care unit patients: A meta-analysis of randomized, placebo-controlled trials. Crit Care Med 2006;34:1216-24.

16. Ho KM, Lipman J, Dobb GJ, Webb SA. The use of prophylactic fluconazole in immunocompetent high-risk surgical patients: A meta-analysis. Crit Care 2005;9:R710-7.

17. Muskett H, Shahin J, Eyres G, Harvey S, Rowan K, Harrison D. Risk factors for invasive fungal disease in critically ill adult patients: A systematic review. Crit Care 2011;15:R287.

18. Pittet D, Monod M, Suter PM, Frenk E, Auckenthaler R. Candida colonization and subsequent infections in critically ill surgical patients. Ann Surg 1994;220:751-8.

19. Dupont H, Bourichon A, Paugam-Burtz C, Mantz J, Desmonts JM. Can yeast isolation in peritoneal fluid be predicted in intensive care unit patients with peritonitis? Crit Care Med 2003;31:752-7.

20. Michalopoulos AS, Geroulanos S, Mentzelopoulos SD. Determinants of candidemia and candidemia-related death in cardiothoracic ICU patients. Chest 2003;124:2244-55.

21. Paphitou NI, Ostrosky-Zeichner L, Rex JH. Rules for identifying. patients at increased risk for candidal infections in the surgical intensive care unit: Approach to developing practical criteria for systematic use in antifungal prophylaxis trials. Med Mycol 2005;43:235-43.

22. Ostrosky-Zeichner L, Sable C, Sobel J, Alexander BD, Donowitz G, Kan V, et al. Multicenter retrospective development and validation of a clinical prediction rule for nosocomial invasive candidiasis in the intensive care setting. Eur J Clin Microbiol Infect Dis 2007;26:271-6.

23. Ostrosky-Zeichner L, Pappas PG, Shoham S, Reboli A, Barron MA, Sims C, et al. Improvement of a clinical prediction rule for clinical trials on prophylaxis for invasive candidiasis in the intensive care unit. Mycoses 2011;54:46-51.

24. Shorr AF, Tabak YP, Johannes RS, Sun X, Spalding J, Kollef MH. Candidemia on presentation to the hospital: Development and validation of a risk score. Crit Care 2009;13:R156.

25. Harrison D, Muskett H, Harvey S, Grieve R, Shahin J, Patel K, et al. Development and validation of a risk model for identification of non-neutropenic, critically ill adult patients at high risk of invasive Candida infection: The Fungal Infection Risk Evaluation (FIRE) Study. Health Technol Assess 2013;17:1-156.

26. Hermsen ED, Zapapas MK, Maiefski M, Rupp ME, Freifeld AG, Kalil AC. Validation and comparison of clinical prediction rules for invasive candidiasis in intensive care unit patients: A matched case-control study. Crit Care 2011;15:R198.

27. Playford EG, Lipman J, Kabir M, McBryde ES, Nimmo GR, Lau A, et al. Assessment of clinical risk predictive rules for invasive candidiasis in a prospective multicentre cohort of ICU patients. Intensive Care Med $2009 ; 35: 2141-5$

28. León C, Ruiz-Santana S, Saavedra P, Almirante B, Nolla-Salas J, Alvarez-Lerma F, et al. A bedside scoring system ("Candida score") for early antifungal treatment in nonneutropenic critically ill patients with Candida colonization. Crit Care Med 2006;34:730-7.

29. León C, Ruiz-Santana S, Saavedra P, Galván B, Blanco A, Castro C, et al. Usefulness of the "Candida score" for discriminating between Candida colonization and invasive candidiasis in non-neutropenic critically ill patients: A prospective multicenter study. Crit Care Med 2009;37:1624-33.

30. Leroy G, Lambiotte F, Thévenin D, Lemaire C, Parmentier E, Devos P, et al. Evaluation of "Candida score" in critically ill patients: A prospective, multicenter, observational, cohort study. Ann Intensive Care 2011;1:50.

31. Hall AM, Poole LA, Renton B, Wozniak A, Fisher M, Neal T, et al. Prediction of invasive candidal infection in critically ill patients with severe acute pancreatitis. Crit Care 2013;17:R49.

How to cite this article: Ahmed A, Azim A, Baronia AK, Marak KS, Gurjar M. Risk prediction for invasive candidiasis. Indian J Crit Care Med 2014;18:682-8.

Source of Support: Nil, Conflict of Interest: None declared. 